\title{
Interlaboratory Program to Characterize Discrete Second-Phase Particles by Image Analysis
}

\author{
George F. Vander Voort
}

Buehler Ltd, 41 Waukegan Road, Lake Bluff, IL 60044

An interlaboratory "round robin" test program was conducted to determine the problems associated with the characterization of discrete second-phase particles according to ASTM E 1245 using automated image analysis. Images were captured from nine specimens, which were given to the eleven participating laboratories. The eleven participants reside in five different countries and most used different image analysis programs. There were two people using one system and another two using a different system. The remaining seven laboratories used different systems. The volume fraction of the second-phase particles varied from about 2 to $25 \%$. Six specimens contained volume fractions from 2 to $3 \%$. For the low volume fraction specimens, 25 fields were captured randomly for five specimens and 15 fields for the sixth specimen. For the other three specimens, either 15 or 10 fields were captured. For actual measurement work, a greater number of fields should be evaluated to obtain good statistical precision.

This was a preliminary round robin and, therefore, no instructions were given to the participants except what to measure. This approach is guaranteed to reveal many problems in developing a standard test method, some of which might not be expected. However, this approach does not yield the best information about the potential precision of a well-defined test method, but it may detect factors that would create bias. The data is analyzed using ASTM E 691, "Standard Practice for Conducting an Interlaboratory Study to Determine the Precision of a Test Method." Although this analysis procedure was performed, since it was a preliminary round robin, the results will not be discussed. Instead, the discussion will focus on the problems encountered in running the program (other than getting people to volunteer and actually do the work, a major problem).

The participants were told to determine the area fraction, $A_{A}$, of the particles, their number per unit area, $\mathrm{N}_{\mathrm{A}}$, their average area, $\mathrm{A}$, and average length, $\mathrm{L}$, and the mean free path, that is, the mean edgeto-edge spacing between particles in all directions. These measurements involve 0-, 1- and 2dimemsional measurements and some calculations. As might be expected, the precision of a 0 -order measurement is usually better than a 1-dimensional measurement, which is usually better than a 2dimensional measurement. The area fraction is simply the ratio of the number of detected pixels to the total number of pixels, without any need to perform any corrections to the image. As a 0dimensional measurement, it should exhibit the best precision. The average particle length is a 1dimensional measurement while the average particle area is a 2-dimensional measurement. Hence, $\mathrm{L}$ should exhibit better precision than A. The number per unit area measurement requires corrections as some particles will intersect the field borders and would be counted twice. There are several ways to do this. In one approach, a guard frame is placed inside the field boundaries with a spacing of about the size of the particles. Then, those particles that intersect two of these borders are counted while those that intersect the other two are not counted. The test area is now the area defined by the guard frame, rather than the original field border. Early image analyzers had counting logic problems in that, depending upon the particle shape, some particles would be counted incorrectly, e.g., instead of counting a particle as 1 , it might be counted as -1 or 2 . Very small particles cannot be sized 
accurately and no instructions were given regarding deletion of such particles from the analysis. Also, in the higher volume fraction specimen, many touching particles were present and no instructions were given about this problem. The round robin used stored images and did not test specimen preparation or specimen inhomogeneity, two factors that certainly influence precision and bias, but are different than characterization variables.

Table 1 is a summary of the mean $\%$ relative accuracy, \%RA, of all the data from each specimen. The $\%$ RA is the $95 \%$ confidence interval divided by the mean expressed as a percentage. Note that specimens 5 and 6 had the highest \% RA values. The particles in specimen 5 were sigma phase detected in 329 stainless steel and the image contrast was poorer than for the other specimens. Specimen 6 was delta ferrite in 304 stainless steel and the image contrast was better, but only 15 fields were captured while 1 through 5 had 25 fields, thus giving them better precision at the same approximate area fraction. Specimen 7 had the best \%RA and was delta ferrite in 15-7PH stainless steel with an area fraction of over $18 \%$ and 15 captured fields. Specimens 8 and 9 were taken from the same composite and the variation in the data represented inhomogeneity in the structure and imprecision in the measurements. Table 2 lists the mean \% RA values for the five measurements made on all of the specimens. Note that the best \%RA is obtained with the $A_{A}$, and the \%RA for the $\mathrm{L}$ values are better than for the A values, as expected. The $\mathrm{N}_{\mathrm{A}}$ values have a high $\% \mathrm{RA}$, probably due mainly to variations in handling very small particles or particles at the boundaries. The MFP data is slightly misleading as three laboratories reported erroneous data, two were exceptionally low and one was exceptionally high. The writer had to contact the laboratories and get these corrected. The MFP is based upon the $A_{A}$ and $N_{L}$, the number of particles intercepted by test lines (rows and columns of pixels), and the users misinterpreted what the intercept count data represented.

Table 1. Average \% Relative Accuracies, for All Measurements, by Specimen

\begin{tabular}{|l|l|l|l|l|l|l|l|l|l|}
\hline Specimen & 1 & 2 & 3 & 4 & 5 & 6 & 7 & 8 & 9 \\
\hline $\begin{array}{l}\text { Avg. } \\
\text { \%RA }\end{array}$ & 10.18 & 12.68 & 13.24 & 12.44 & 14.9 & 14.78 & 7.53 & 13.44 & 12.66 \\
\hline
\end{tabular}

Table 2. Average \% Relative Accuracies, for All Specimens, by Measurement

\begin{tabular}{|l|l|l|l|l|l|}
\hline Measurement & $\mathrm{A}_{\mathrm{A}}$ & $\mathrm{N}_{\mathrm{A}}$ & $\mathrm{A}$ & $\mathrm{L}$ & MFP \\
\hline Avg. \% RA & 8.47 & 14.39 & 15.93 & 13.68 & 9.68 \\
\hline
\end{tabular}

The program revealed numerous problems. For the individual data analysis, some labs averaged the $\mathrm{A}$ and $\mathrm{L}$ data in each field and used the field averages to compute the mean and standard deviation. Others determined the mean and standard deviation on all of the data, which yields a significantly higher s value and a somewhat different mean. This difference makes running the E 691 statistical analysis difficult as the labs with the correct high s value are deemed as outliers. Small particles, e.g., those comprised of 5 or fewer pixels, should be deleted from the analysis for area and length, as the errors will be substantial. Touching particles must be separated, e.g., by an ultimate erosion and reconstruction method. The same procedure should be used to correct for particles at the field borders. Intercept counting is not offered by all systems, and when available, the manual must clearly state exactly what the output represents. These and other results will be used to fully define the test variables and then another round robin will be conducted. 